\title{
Adult Anopheles Mosquito Distribution at a Low and High Malaria Transmission Site in Tanzania
}

\author{
David Zadock Munisi $\mathbb{C}^{1}$ and Mary Mathew Mathania $\mathbb{D}^{2}$ \\ ${ }^{1}$ Department of Microbiology and Parasitology, School of Medicine and Dentistry, The University of Dodoma, P.O. Box 259, \\ Dodoma, Tanzania \\ ${ }^{2}$ Department of Basic and Behavioral Sciences, School of Nursing, Saint John's University of Tanzania, Dodoma, Tanzania
}

Correspondence should be addressed to David Zadock Munisi; massugii@gmail.com

Received 4 September 2021; Accepted 11 December 2021; Published 10 January 2022

Academic Editor: Praveen Bharti

Copyright (C) 2022 David Zadock Munisi and Mary Mathew Mathania. This is an open access article distributed under the Creative Commons Attribution License, which permits unrestricted use, distribution, and reproduction in any medium, provided the original work is properly cited.

\begin{abstract}
Malaria parasites are only transmitted by female mosquitoes of the genus Anopheles; hence, the disease's distribution is linked to that of the vector mosquitoes. As such, the goal of this study was to find out the spatial and temporal distribution of Anopheles mosquito adults in the research sites. This was a repeated cross-sectional ecological study that took place in Morogoro and Dodoma, Tanzania. Vacuum aspiration was used to collect mosquitoes both outside and inside human dwellings. All mosquito-related data was collected and entered into appropriate data collection forms. Female mosquitoes were recognized morphologically using Gillies and Coetzee morphological criteria, followed by PCR. In total, about 2742 Anopheles mosquitoes with an average collection of $18.21 \pm 1.12$ per day were collected outside human houses of which $1717(10.51 \pm 1.17)$ and 1025 $(8.42 \pm 1.41)$ were collected from Morogoro and Dodoma, respectively. Of the captured mosquitoes, $89.0 \%, 10.0 \%$, and $1.0 \%$ were recognized as Anopheles arabiensis, Anopheles gambiae s.s., and Anopheles quadrianulatus, respectively. The distribution varied significantly with seasons, whereby $302(4.72 \pm 1.04)$ and $2440(12.96 \pm 1.52)$ mosquitoes were captured in the cold-dry and warm-wet season, respectively $(p<0.0001)$. Of the captured mosquitoes, $42.33 \%, 16.33 \%, 14.96 \%$, and 4.27 were found on the ceiling, stored junks, verandas, and barks/tree, respectively. In malaria-endemic countries, vector control forms an important component of the malaria control efforts. This study found significant variation of Anopheles mosquito abundance in time and space with Anopheles arabiensis being the most predominant malaria vector. This signifies the need to introduce mosquito control methods that will target the less anthropophilic Anopheles arabiensis or the immature aquatic stages. The study further found that underbeds, store room/piled bags, and undisturbed curtains were the most preferred resting places by mosquitoes signifying to be the most effective strategic sites for spraying insecticides during the implementation of indoor residual spraying (IRS).
\end{abstract}

\section{Introduction}

Malaria is a major public health issue in Tanzania, with 93 percent of the population being at risk, the disease is the main national cause of outpatient visits and admissions in health care facilities, particularly among children under the age of five and pregnant women [1]. The disease is caused by protozoan parasites of the genus Plasmodium which are exclusively transmitted by female Anopheles mosquitoes [2]; consequently, the distribution of the disease correlates with the distribution of the vector mosquitoes [3].
Macro (temperature and topography) and micro (availability of aquatic biological niches) factors influence the occurrence of vector Anopheles mosquitoes. Within a district, region, country, or continent, climate and topography are significant factors of macro spatial-temporal Anopheles mosquito distribution [3-6]. The availability of aquatic habitats close to human dwellings determines the micro -spatial occurrence of vector mosquitoes within the community [7, 8]. Adult mosquito distributions are typically seasonal and follow rainfall patterns, resulting in population densities differing between and within countries [9]. The knowledge of 
malaria transmission dynamics, particularly the temporal and spatial distribution of Anopheles mosquito vector populations, is generally low due to Tanzania's large geographic size and different ecoclimatic conditions. It is widely acknowledged that vector control plays a critical role in achieving malaria elimination in endemic areas $[10,11]$. Understanding the temporal and spatial distribution of sympatric malaria vector species is important for effective vector control in malaria-endemic countries like Tanzania $[2,12]$. This research intended, therefore, to investigate distribution in time and space of Anopheles mosquito adults so as to have a better understanding of the distribution of these important malaria vectors to be used in implementing different vector control measures that target adult mosquitoes.

\section{Methodology}

2.1. Study Area. The research was carried out in Tanzania's Dodoma and Morogoro regions. Morogoro has a high malaria prevalence of 9.5 percent, while Dodoma has a low malaria prevalence of 0.6 percent [13]. The Morogoro municipality is located in Tanzania's eastern region, about 169 kilometers west of Dar-es-Salaam and about 223 kilometers east of Dodoma, the country's capital. Short rains fall in December and January, and long rains fall from March through June. During the rainy seasons (December-May), the mean annual rainfall is about $783.5 \mathrm{~mm}$, with a mean relative humidity of about 72 percent, a lowest temperature of $22^{\circ} \mathrm{C}$, and a high temperature of $33^{\circ} \mathrm{C}$ during the wet season. The minimum and maximum temperatures throughout the cold season (June-September) are $15^{\circ} \mathrm{C}$ and $19^{\circ} \mathrm{C}$, respectively. Dodoma is 486 kilometers west of Dar-es-Salaam, located at $6^{\circ} 25^{\prime} \mathrm{S}$ and $35^{\circ} 75^{\prime} \mathrm{E}$. The study areas have been described in details in our earlier publication [14].

2.2. Study Design. The research was a repeated crosssectional ecological study. The data was gathered throughout two seasons: the cold-dry season from June to September 2014 and the hot-wet season from January to February 2015. Adult mosquitoes were collected in the same areas inside and outside human houses during both seasons of the study.

2.3. Sampling Techniques. Purposefully, two wards were chose both in Dodoma and Morogoro urban on the basis of their having seasonal and temporary breeding habitats (presence of artificial breeding sites, natural wetlands, and rice fields) as well as a local high incidence of malaria [15, $16]$.

\subsection{Data Collection Procedure}

2.4.1. Data Collection on Adult Mosquito. From the sampled wards, ten houses were randomly selected from each street in Morogoro and Dodoma. A handheld global positioning system (GPS) device was used to map out the homes that were included in the mosquito sampling. Mosquitoes were collected using a vacuum backpack aspirator outside and inside human dwellings in places like verandas, unfinished buildings (inside and out), fences, stored junks, and tree holes, as well as behind curtains, underbeds, and under couches, while knockdown catches were done in places like storerooms with pyrethrum aerosol spray (from Rungu ${ }^{\circledR}$ aerosol spray cans). The catching took place in the mornings between 0700 and $1000 \mathrm{hrs}$ and in the evenings between 1700 and 1900 hrs. All mosquito-related data was filled into the appropriate data collection forms. [17]. A vacuum aspirator with a collection cup at the back was used to gather mosquitoes trapped in the field during mosquito collection. The mosquitoes in the collecting cup were emptied into transportation plastic cups (labeled with street names) and moved to the field before being counted and sorted at the insectary. The morphological keys of Gillies and Coetzee were used to identify female mosquitoes. Following morphological identification, mosquitoes were analyzed to species siblings by PCR.

\subsubsection{Differentiation of Anopheles Species by Polymerase} Chain Reaction Analysis. PCR was used to identify a total of 100 mosquitoes that were randomly selected from a total of 2742 which were morphologically identified to be adult female Anopheles mosquitoes. All of the female Anopheles that were chosen were taken to the lab for DNA extraction and PCR analysis. The DNA extraction protocol is as described in our earlier publication [14]. The isolated DNA pellet was kept at $-20^{\circ} \mathrm{C}$ until it was used to conduct the PCR analysis.

PCR techniques developed by Scott et al. [18] were used to differentiate the Anopheles gambiae complex. The PCR amplification for the Anopheles gambiae complex was carried out using universal and species-specific primers, as described in our previous publication [14]. The speciesspecific nucleotide sequences in the ribosomal DNA intergenic spacer (IGS) were used to identify the An. gambiae complex using three deferentially sized amplicons. Anopheles gambiae s.s. (390 bp), Anopheles arabiensis (315 bp), and Anopheles quadriannulaus $(150 \mathrm{bp})$ were the species extracted product sizes [18]. A UV transilluminator was used to visualize the amplified DNA on a 2.0 percent agarose gel stained with ethidium bromide. The primers for the Anopheles gambiae complex are listed below [14]:

\section{Primers}

UN: $5^{\prime}$-GTG TGC CCC TTC CTC GAT GT-3'

GA: $5^{\prime}$-CTG GTT TGG TCG GCA CGT TT-3'

AR: $5^{\prime}$-AAG TGT CCT TCT CCA TCC TA-3'

QD: $5^{\prime}$-CAG ACC AAG ATG GTT AGT AT-3'

2.4.3. Establishment of Sheltering Places of Vector Anopheles Mosquitoes during Dry-Cold Season (June-September). From June to September 2015, researchers conducted an identification of mosquitoes hiding places in randomly selected houses in each study site (ten houses each in Morogoro and Dodoma). Mosquitoes were collected using spray sheet collection method with knockdown catches using pyrethrum aerosol spray (from Rungu ${ }^{\circledR}$-aerosol spray cans) in places like storerooms, while an aspirator was used to collect mosquitoes in undisturbed places like under the bed, store rooms, behind the curtains, shelves, and ceilings. Following that, all mosquitoes caught in aspirator cups and knockdown 
catches (fell on the white sheet) were temporarily placed in labelled carrying plastic cups before being counted; they were then sorted by sex and identified morphologically in the laboratory. Each female Anopheles mosquito was maintained in an Eppendorf filled with silica gel after morphological identification. The silica gel was employed to keep mosquitoes dry as they waited to be killed.

2.5. Data Processing and Analysis. To investigate the impact of research site variables on Anopheles mosquito distribution, data on the number of vector mosquitoes collected in various locations were modelled using a Poisson regression model. Mosquitoes were collected and taken to the laboratory for morphological and molecular analysis. As indicated in General Laboratory Analysis, identified vector mosquitoes were sampled and submitted for PCR identification.

2.6. Ethical Considerations. The Sokoine University of Agriculture's (SUA) Directorate of Research and Postgraduate Studies provided ethical approval for the research. The district and local ward administrations provided permission to conduct the study. After presenting the study's objectives to the head of the household, written consent was acquired to set up the trials in selected households to evaluate the Umbrella-topped Contaminating Device (UtMCD).

\section{Results}

3.1. Adult Anopheles Mosquito Distribution Based on Study Characteristics. Outside human residences, a total of 2742 Anopheles mosquitoes were captured, with an average of $18.21 \pm 1.12$ per day, with $1717(10.51 \pm 1.17)$ being collected in Morogoro. Only $302(4.72 \pm 1.04)$ were captured during the dry-cold season (June-September), while 2440 $(12.96 \pm 1.52)$ were collected during the wet-hot season (March-May). The quantity of collected mosquitoes from different sites was analyzed, and Mahita in Morogoro (621 $(21.64 \pm 2.74))$ and Swaswa in Dodoma (576 (18.87 \pm 2.59 )) had the largest quantities of mosquitoes collected (Table 1).

3.2. Adult Anopheles Mosquito Distribution in Different Sites. Out of 2742 of vector Anopheles mosquitoes caught, majority $(42.33 \%)$ were found on the ceiling, followed by $16.33 \%$, $14.96 \%$, and $4.27 \%$ in stored junks, verandas, and barks/tree, respectively (Figure 1). The variation in the distribution of vector mosquitoes by location was observed to be statistically significant with respect to season $\left(\chi^{2}=168.79, p=<\right.$ 0.0001 ), whereby in dry-cold season (June-September), a total of 320 vector Anopheles mosquitoes were caught outside houses of which $36.47 \%, 22.43 \%, 16.11 \%$, and $8.55 \%$ were collected from ceiling, stored junks, verandas, and inside unfinished buildings, respectively. In wet-warm season (March-May), out of 2440 Anopheles mosquitoes caught, the highest percent $(49.03 \%)$ was found on ceiling followed by $13.64 \%, 9.35 \%$, and $9.28 \%$ in verandas, stored junks, and fence/flower, respectively (Figure 2). To determine the effect of season on the distribution of the collected adult mosquitoes, a Poisson regression model was constructed. The model's results, which are presented in
TABle 1: Adult Anopheles mosquito distribution based on study characteristics.

\begin{tabular}{lcc}
\hline Variable & Number & Mean \pm SE \\
\hline Region & 1717 & $10.51 \pm 1.17$ \\
Morogoro & 1025 & $8.42 \pm 1.41$ \\
Dodoma & & \\
\hline Season & 302 & $4.72 \pm 1.04$ \\
Dry & 2440 & $12.96 \pm 1.52$ \\
Wet & & \\
\hline Area & & \\
Morogoro & 272 & $7.20 \pm 1.54$ \\
Tupendane & 164 & $8.37 \pm 1.47$ \\
Visegese & 621 & $21.64 \pm 2.74$ \\
Mahita & 447 & $13.55 \pm 2.15$ \\
Mwembeni & 213 & $11.65 \pm 2.07$ \\
Misufini & & \\
Dodoma & 449 & $18.97 \pm 2.59$ \\
Mnarani & 576 & \\
Swaswa & &
\end{tabular}

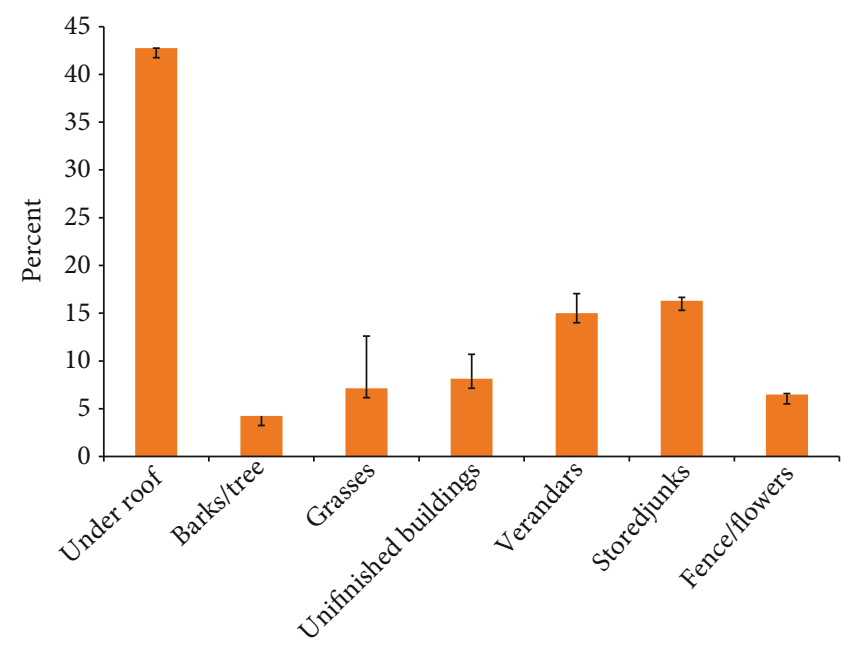

Figure 1: Adult Anopheles mosquito percentage distribution by location. Standard error of the mean is represented by error bars.

Table 2, show that after adjusting for the study area, the distribution of adult mosquitoes was significantly associated with season $(p<0.0001)$, with the mean number of mosquitoes collected during the cold-dry season (June-September) being nearly twice as much to that collected during the rainy season (March-May) (AMR $=1.6, p<0.0001)$. Furthermore, the average number of adult mosquitoes collected in Morogoro $(\mathrm{AMR}=1.34, p<0.0001)$ was significantly higher than that in Dodoma $(\mathrm{AMR}=1.34, p<0.0001)$ (Table 2).

3.3. The Species Composition of Adult Mosquitoes Collected. PCR was used to identify 100 mosquitoes randomly picked from a total of 2742 morphologically identified adult female 


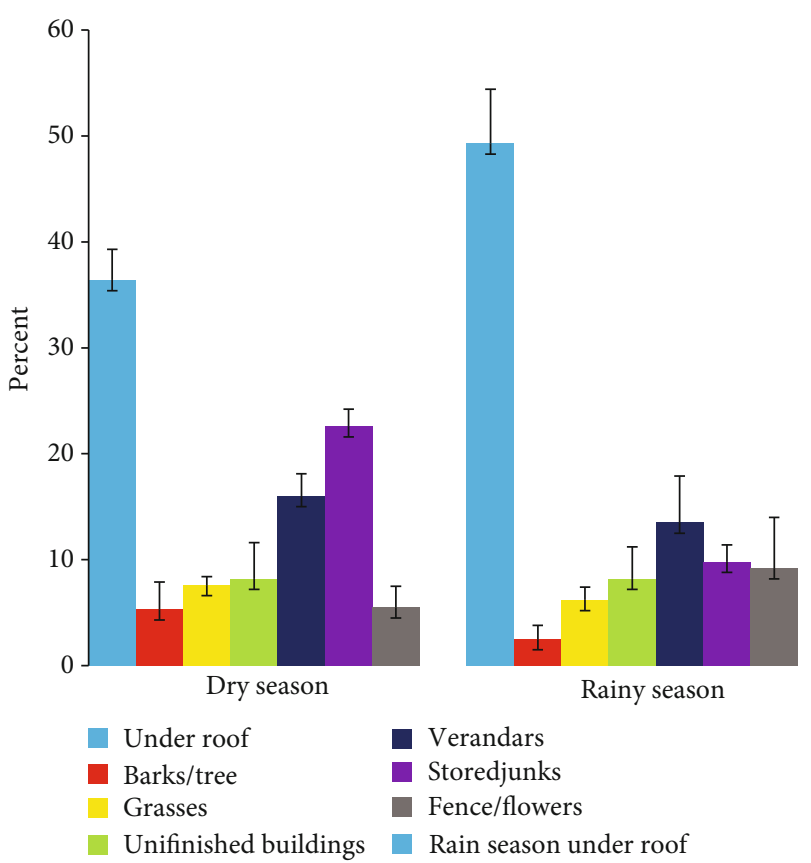

Figure 2: Adult mosquito distribution by season and place as a percentage. The standard error of the mean is represented by the error bars.

TABle 2: The Poisson regression model's parameter estimates, adjusted mean ratio (AMR), and significance levels for the effect of region and season on adult mosquito distribution.

\begin{tabular}{lcccc}
\hline Parameter & Estimate $(\widehat{\beta})$ & SE & $p$ value & AMR \\
\hline $\begin{array}{l}\text { Intercept } \\
\text { Region }\end{array}$ & 2.470 & 0.040 & $<0.0001$ & 11.85 \\
Morogoro & 0.290 & 0.040 & $<0.0069$ & 1.34 \\
$\begin{array}{l}\text { Dodoma } \\
\text { Season }\end{array}$ & 1 & & & \\
Dry & 0.470 & 0.030 & $<0.0001$ & 1.60 \\
Rain & 1 & & & \\
\hline
\end{tabular}

Anopheles mosquitoes. Anopheles arabiensis accounted for $89.0 \%$ of the total, whereas Anopheles gambiae s.s. accounted for $10.0 \%$, and Anopheles quadrianulatus accounted for only1.0\%.

3.4. Anopheles Mosquitoes' Sheltering Places during Cold-Dry Seasons. During the cold-dry season (June-September), 1541 mosquitoes were collected inside human dwellings, with an average daily collection of $19.26 \pm 1.81$. Mosquitoes have been found to take up residence in various locations within homes. According to the data, Dodoma had the highest mean number of collected mosquitoes from sheltering locations $(26.63 \pm 2.37)$, with Swaswa street having the highest mean Anopheles catches $(32.07 \pm 3.33)$ (Table 3$)$.

3.5. Anopheles Mosquito's Percentage Distribution according to Sheltering Places. Of all the collected mosquitoes (1541), the majority $(32.06 \%)$ were collected underbeds, followed
TABLE 3: Anopheles mosquito sheltering in different sites in Dodoma and Morogoro, mean distribution.

\begin{tabular}{lc}
\hline Variable & Mean \pm SE \\
\hline Region & $14.84 \pm 1.03$ \\
Morogoro & $26.63 \pm 2.37$ \\
Dodoma & \\
Study area (streets) & \\
Morogoro & $12.80 \pm 1.81$ \\
Mwembeni & $12.40 \pm 1.52$ \\
Visegese & $14.60 \pm 1.93$ \\
Tupendane & $15.40 \pm 1.98$ \\
Misufini & $17.00 \pm 2.39$ \\
Mahita & \\
Dodoma & $21.20 \pm 2.08$ \\
Mnalani & $32.07 \pm 3.33$ \\
Swaswa &
\end{tabular}

by $25.31 \%, 23.75 \%$ and $4.48 \%$ behind undisturbed curtains, store room/piled bags, and ceilings, respectively (Figure 3).

3.6. Poisson Regression Model for the Effect of Region on Mosquito Distribution. The Poisson regression model (Table 4) for the effect of region on the distribution of Anopheles mosquitoes during the sheltering period in dry season revealed that the average number of vector Anopheles mosquitoes captured in Morogoro $(\mathrm{MR}=0.56, p=$ 0.0002 ) was significantly lower than that captured in Dodoma.

\section{Discussion}

Vector control forms an important part of malaria control program. Effective vector control is dependent on a good understanding of the distribution of the vector mosquitoes in endemic communities [2, 10-12]. We report the distribution of vector Anopheles mosquito as determined in the two study areas.

Significant variation on adult mosquito abundance between places has been reported in other places, as a possible explanations, availability of favourable larval habitats, the distance from residents or houses to larval habitats, and the types of house roofs have been postulated [19]. Similarly, our study reports not only a relatively higher numbers of mosquitoes in the region known to have higher malaria prevalence, i.e., Morogoro as compared to Dodoma, but also expectedly, mosquitoes were plentiful during rainy season than during cold/dry season owing to the abundancy of vector larval breeding sites in wet/hot season than in the other season. The observed variation of mosquito abundance in time was mainly as a result of rainfall changes in different seasons. Anopheles mosquito abundance may be used as a proxy of malaria transmission intensity and therefore used to guide focused malaria control interventions in endemic areas as countries move towards malaria focal elimination. As already known, the distribution of malaria can be quite 


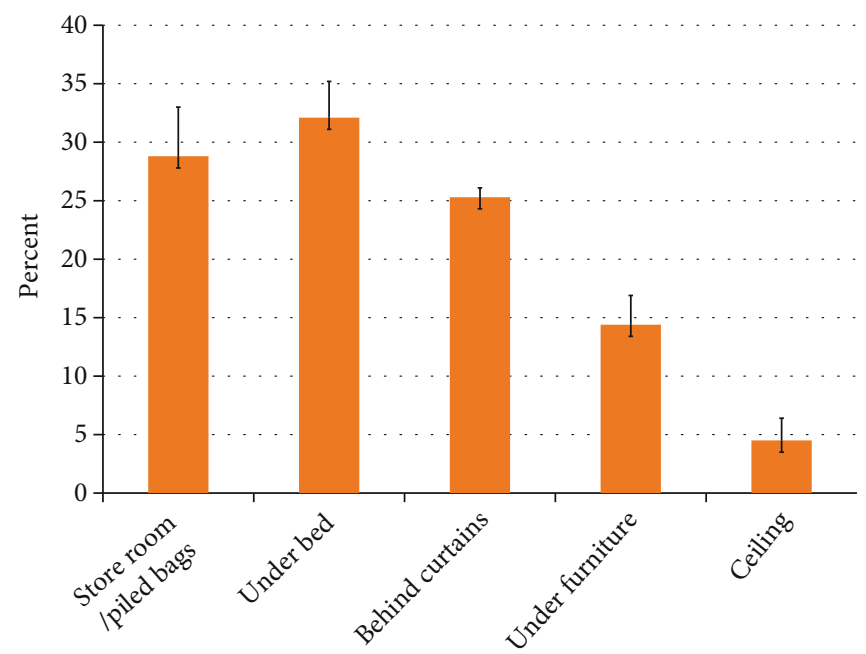

FIgURe 3: Anopheles mosquitoes' percentage distribution according to sheltering places inside human houses. Error bars indicate standard error $(n=1541)$.

TABle 4: Poisson regression model's parameter estimates and adjusted mean ratio (MR) for the effect of region on distribution of sheltering Anopheles mosquitoes collected during dry-cold season.

\begin{tabular}{lcccc}
\hline Parameter & Estimate $(\widehat{\beta})$ & SE & $p$ value & MR \\
\hline $\begin{array}{l}\text { Intercept } \\
\text { Region }\end{array}$ & 3.280 & 0.110 & $<0.0001$ & 26.630 \\
$\begin{array}{l}\text { Morogoro } \\
\text { Dodoma }\end{array}$ & -0.580 & 0.160 & 0.0002 & 0.560 \\
\hline
\end{tabular}

variable even over very short distances within the same village; in our study, this was evident as we found high mosquito abundance in Mahita and Swaswa in Morogoro and Dodoma, respectively, as compared to other parts. Knowledge of this spatial distribution of Anopheles mosquito vector dictated by certain environmental, climatic, and anthropogenic factors can be deployed when defining residual malaria transmission while advancing to malaria elimination.

Anopheles arabiensis was the main malaria vector in the research area during both seasons. The use of LLINs/ITNs and IRS, which reduce the extremely anthropophilic Anopheles species Anopheles gambiae s.s., which is now being replaced by Anopheles arabiensis, is most likely the reason for the increasing abundance of Anopheles arabiensis [12]. In different places in Tanzania, it has been observed that there is a significant shift in the Anopheles mosquito abundance from a predominantly Anopheles gambiae s.s. to Anopheles arabiensis [12, 20-22]. This has also been observed in the study area especially Morogoro where in previous years, Anopheles gambiae s.s. were the most wide spread species but as observed in our study, the surveyed wards had over $80 \%$ of collected Anopheles being Anopheles arabiensis [20,21].

Expectedly, during the cold-dry season, vector mosquitoes disappear to aestivate/hibernate [23-25]. This study has found that the majority of mosquitoes hide inside build- ings during the dry/cold season in the dark and/or regions with little or no disturbances, whereby underbeds, behind undisturbed curtains, store rooms/stored junks, and under furniture were the most commonly preferred hiding places during the dry/cold season. Merdic and colleagues discovered that during the cold/dry season, vector Anopheles mosquitoes concealed in cellars, deep basements, dark rooms with small windows, and on dirt floors formed of bricks [26]. According to many studies that explain how mosquitoes become immobile during the cold/dry season [23-25, 27], spraying methods that knock down mosquitoes can be used during the cold season to kill many mosquitoes, reducing population density in the community before the warm/ wet seasons when mosquitoes emerge from hiding places. However, in Tanzania, indoor residual spraying (IRS) is a malaria management approach that targets adult mosquito vectors [28]. A good understanding of the resting behavior of local mosquitoes is required for an efficient indoor residual spraying (IRS) campaign. According to the findings of our study, spraying underbeds, storage rooms/piled bags, and undisturbed curtains is likely to accomplish greatest mosquito control, as these are mosquitoes' preferred resting spots.

\section{Conclusion}

Vector control is an important component of malaria control in endemic countries. A good understanding of the spatial-temporal distribution of the Anopheles mosquito vector forms a necessary prerequisite in developing a sound and effective vector control program. This study found significant variation of Anopheles mosquito population in time and space with Anopheles arabiensis being the most predominant malaria vector found. The predominance of Anopheles arabiensis which is a zooanthropohilic mosquito as compared to the highly anthropohilic Anopheles gambiae signifies success of LLINs/ITNs and IRS. However, this also signifies the need to introduce methods in malaria control 
arsenal of methods that will also kill the less anthropophilic Anopheles arabiensis or methods directed towards the immature aquatic stages which will indiscriminately kill all Anopheles larva. The study further found that underbeds, store room/piled bags, and undisturbed curtains were the most preferred resting places by mosquitoes signifying to be the most effective strategic sites for spraying insecticides during the implementation of IRS.

\section{Data Availability}

Data underlying this study are available upon request.

\section{Conflicts of Interest}

The authors declare that they have no competing interests.

\section{Acknowledgments}

The authors extend their gratitude to the Intra-ACP Academic Mobility Scheme for the financial support to complete the study.

\section{References}

[1] WHO, "WHO recognizes national efforts towards Malaria elimination," in WHO recognizes national efforts towards Malaria elimination, U. R. Tanzania, Ed., WHO, Dar es salaam, 2018, https://afro.who.int/news/who-recognizesnational-efforts-towards-malaria-elimination.

[2] L. A. Kelly-Hope, J. Hemingway, and F. E. McKenzie, "Environmental factors associated with the malaria vectors Anopheles gambiae and Anopheles funestus in Kenya," Malaria Journal, vol. 8, no. 1, 2009.

[3] K. C. Ernst, S. O. Adoka, D. O. Kowuor, M. L. Wilson, and C. C. John, "Malaria hotspot areas in a highland Kenya site are consistent in epidemic and non-epidemic years and are associated with ecological factors," Malaria Journal, vol. 5, p. 78, 2006.

[4] K. L. Gage, T. R. Burkot, R. J. Eisen, and E. B. Hayes, "Climate and vectorborne diseases," American Journal of Preventive Medicine, vol. 35, pp. 436-450, 2008.

[5] M. A. Kulkarni, R. E. Desrochers, and J. T. Kerr, "High resolution niche models of malaria vectors in northern Tanzania: a new capacity to predict malaria risk?," PLoS One, vol. 5, no. 2, article e9396, 2010.

[6] G. Texier, V. Machault, M. Barragti, J.-P. Boutin, and C. Rogier, "Environmental determinant of malaria cases among travellers," Malaria Journal, vol. 12, 2013.

[7] W. Gu, J. Utzinger, and R. J. Novak, "Habitat-based larval interventions: a new perspective for malaria control," The American Journal of Tropical Medicine and Hygiene, vol. 78, no. 1, pp. 2-6, 2008.

[8] Y. Geissbühler, K. Kannady, P. P. Chaki et al., "Microbial larvicide application by a large-scale, community-based program reduces malaria infection prevalence in urban Dar Es Salaam, Tanzania," PLoS One, vol. 4, no. 3, p. e5107, 2009.

[9] M. J. Oesterholt, T. J. Bousema, O. K. Mwerinde et al., "Spatial and temporal variation in malaria transmission in a low endemicity area in northern Tanzania," Malaria Journal, vol. 5, no. $1,2006$.
[10] B. M. Greenwood, "Control to elimination: implications for malaria research," Trends in Parasitology, vol. 24, no. 10, pp. 449-454, 2008.

[11] K. Mendis, A. Rietveld, M. Warsame, A. Bosman, B. Greenwood, and W. H. Wernsdorfer, "From malaria control to eradication: the WHO perspective," Tropical Medicine and International Health, vol. 14, no. 7, pp. 802-809, 2009.

[12] T. L. Russell, N. J. Govella, S. Azizi, C. J. Drakeley, S. P. Kachur, and G. F. Killeen, "Increased proportions of outdoor feeding among residual malaria vector populations following increased use of insecticide-treated nets in rural Tanzania," Malaria Journal, vol. 10, 2011.

[13] Ministry of Health, Community Development, Gender, Elderly and Children (MoHCDGEC) [Tanzania Mainland], Ministry of Health $(\mathrm{MoH})$ [Zanzibar], National Bureau of Statistics (NBS), Office of the Chief Government Statistician (OCGS), and ICF, Tanzania Malaria Indicator Survey, MoHCDGEC, MoH, NBS, OCGS, and ICF, Dar es Salaam, Tanzania, and Rockville, Maryland, USA, 2017.

[14] M. M. Mathania, D. Z. Munisi, and R. S. Silayo, "Spatial and temporal distribution of Anopheles mosquito's larvae and its determinants in two urban sites in Tanzania with different malaria transmission levels," Parasite Epidemiology and Control, vol. 11, p. e00179, 2020.

[15] MTUHA, Prevalence of malaria, upper and lower respiratory infection, gastrointestinal infection and urinary infection in Dodoma region, Ministry of Health, Community Development, Gender, Elderly and Children (MoHCDGEC), 2015

[16] MTUHA, Prevalence of malaria, upper and lower respiratory infection, gastrointestinal infection and urinary infection in Morogoro region, Ministry of Health, Community Development, Gender, Elderly and Children (MoHCDGEC), 2015

[17] M. T. Gillies and M. Coetzee, A supplement to the Anophelinae of Africa south of the Sahara (Afrotropical Region), A Suppl to Anophelinae Africa south Sahara, 1987, May 2019, https:// www.cabdirect.org/cabdirect/abstract/19880590772.

[18] J. A. Scott, W. G. Brogdon, and F. H. Collins, "Identification of single specimens of the Anopheles gambiae complex by the polymerase chain reaction," The American Journal of Tropical Medicine and Hygiene, vol. 49, pp. 520-529, 1993.

[19] G. Zhou, N. Minakawa, A. Githeko, and G. Yan, "Spatial distribution patterns of malaria vectors and sample size determination in spatially heterogeneous environments: a case study in the west Kenyan highland," Journal of Medical Entomology, vol. 41, pp. 1001-1009, 2004.

[20] T. L. Russell, D. W. Lwetoijera, D. Maliti et al., "Impact of promoting longer-lasting insecticide treatment of bed nets upon malaria transmission in a rural Tanzanian setting with preexisting high coverage of untreated nets," Malaria Journal, vol. 9, 2010.

[21] J. Kitau, R. M. Oxborough, P. K. Tungu et al., "Species shifts in the Anopheles gambiae complex: do LLINs successfully control Anopheles arabiensis?,” PLoS One, vol. 7, no. 3, 2012.

[22] Y. A. Derua, M. Alifrangis, K. M. Hosea et al., "Change in composition of the Anopheles gambiae complex and its possible implications for the transmission of malaria and lymphatic filariasis in north-eastern Tanzania," Malaria Journal, vol. 11, p. 188, 2012.

[23] R. M. Robich and D. L. Denlinger, "Diapause in the mosquito Culex pipiens evokes a metabolic switch from blood feeding to sugar gluttony," Proceedings of the National Academy of 
Sciences of the United States of America, vol. 102, pp. 1591215917, 2005.

[24] D. L. Huestis, A. S. Yaro, A. I. Traoré et al., "Seasonal variation in metabolic rate, flight activity and body size of Anopheles gambiae in the Sahel," The Journal of Experimental Biology, vol. 215, pp. 2013-2021, 2012.

[25] T. Lehmann, A. Dao, A. S. Yaro et al., "Seasonal variation in spatial distributions of Anopheles gambiae in a Sahelian village: evidence for aestivation," Journal of Medical Entomology, vol. 51, pp. 27-38, 2014.

[26] E. Merdić and S. Vujičić-Karlo, "Two types of hibernation of Culex pipiens Complex (Diptera: Culicidae) in Croatia," Entomologia Croatica, vol. 9, pp. 71-76, 2006.

[27] A. S. Yaro, A. I. Traoré, D. L. Huestis et al., "Dry season reproductive depression of Anopheles gambiae in the Sahel," Journal of Insect Physiology, vol. 58, pp. 1050-1059, 2012.

[28] L. E. G. Mboera, H. D. Mazigo, S. F. Rumisha, and R. A. Kramer, "Towards malaria elimination and its implication for vector control, disease management and livelihoods in Tanzania," MalariaWorld Journal, vol. 4, 2013. 